\title{
Reduce energy consumption with an advanced eco-routing guidance strategy
}

\author{
Jie Chen, and Maobin $\mathrm{Hu}^{*}$ \\ School of Engineering Science, University of Science and Technology of China, Hefei 230026, P. R. \\ China
}

\begin{abstract}
Route guidance is an efficient method to improve the capacity and efficiency of traffic system. This paper proposes an advanced ecorouting guidance strategy based on the real-time feedback information of both fuel consumption and travel time under the background of intelligent transportation system. Compared with the geographical shortest path strategy (GSP) and the time shortest path strategy (TSP), simulation experiment results show that the new strategy can help save fuel consumption and traffic cost by less detours. The distribution of travel cost among the roads is also provided, which is consistent with analysis. The implementation of the strategy in real cities might greatly reduce the energy consumption for transportation.
\end{abstract}

Keywords: Eco-routing; Fuel consumption; Traffic flow.

\section{Introduction}

Energy consumption by transportation has increased dramatically in this century. A rough estimation shows that transportation consumes $59 \%$ of oil annually world-wide. With the increase of travel demand, congestion becomes more prevailing in most cities. Heavy traffic has brought many negative effects, including traffic delays, rising accident rates, and increasing fuel consumption.

Recent researches indicate that real-time traffic information, which can be collected by intelligent transportation system (ITS) and then fed back to drivers, can play an important role in improving traffic efficiency [1-2]. Many studies have been done regarding the application of real-time traffic information feedback in energy saving and management [34]. For example, Tang et al. and Cui et al. analysed the effects of real-time information feedback strategy on traffic fuel consumption and emission [5-6].

The traffic flow in urban road network becomes more complex, and one of the important factors determining its performance is the route guidance strategy. With the development of ITS, real-time feedback on vehicle fuel consumption becomes available, which can be provided to drivers for route guidance. In this perspective, we propose a new eco-routing guidance strategy considering both travel time and fuel consumption costs.

\footnotetext{
* Corresponding author: humaobin@ustc.edu.cn
} 


\section{Model}

We assume that the urban traffic system is regular Manhattan-like network structure, as shown in Fig.1, the network contains $N \times N$ intersections. Any two neighbouring intersections are connected by two opposite links, and each link is assumed to have one lane. CA model is adopted to divide each lane into $L$ cells, and one vehicle only occupies a cell at each time step. There is a traffic light equipped at each intersection. Each traffic light includes four phases. For each phase, the traffic light is green for one incoming link, and red for the other three incoming links for $T$ time steps. This network model has been developed recently as an efficient way to analyse network traffic system by reflecting various aspects of real world traffic environment [2].

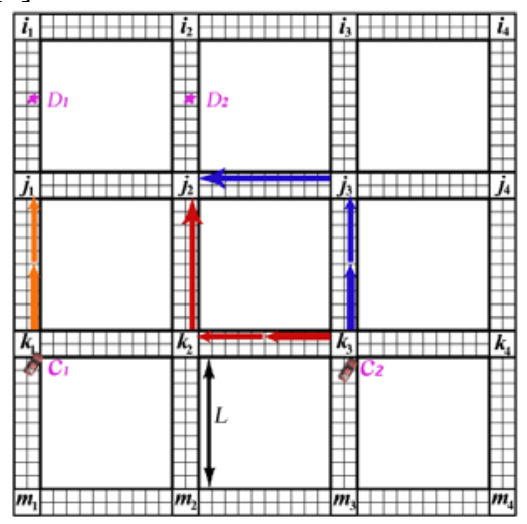

Fig. 1. Schematic illustration of a $4 \times 4$ Manhattan-like road network.

The deterministic Nagel-Schreckenberg (NaSch) rules [7], as one of most popular CA model, is adopted to simulate the movement of vehicles. At each time step, the velocity and location of all vehicles will be updated in parallel as follows:

(1) Acceleration: $v_{i} \rightarrow \min \left(v_{\max }, v_{i}+1\right)$;

(2) Deceleration: $v_{i} \rightarrow \min \left(d_{i}, v_{i}\right)$;

(3) Random brake with probability $p: v_{i}=\max \left(v_{i}-1,0\right)$;

(4) Movement: $x_{i}=x_{i}+v_{i}$.

Here, $v_{\max }$ represents the maximum velocity at which the vehicle can move on the lane. $v_{i}$ and $x_{i}$ are the velocity and location of vehicle $i$, respectively. $d_{i}$ is the number of sequential empty cells ahead of vehicle $i$. Obviously, for the leading vehicle of each lane, $d_{i}$ is determined by the traffic light status and the congestion at the intersection.

We use the comprehensive modal emission model (CMEM) in Refs.[8-9] to estimate fuel consumption of vehicles second-by-second. According to CMEM, the fuel rate (in L/s) is defined as:

$$
f=\frac{\xi}{k \psi}\left\{k N V+\frac{1}{\eta}\left[\frac{(\omega+q) g C_{r} \cos \theta+0.5 C_{d} \rho A v^{2}}{1000 \eta_{t f}} v\right]\right\}
$$

All parameters along with typical values are listed in Table 1.

\section{Route guidance strategy}

GSP strategy: When there is no real-time traffic information is provided, and drivers will choose the paths with the global shortest total travel distance. As shown in Fig.1, vehicles $C_{1}$ and $C_{2}$ are heading to $D_{1}$ and $D_{2}$, respectively. There is one GSP path (indicated by orange 
arrows) for $C_{1}$, and two GSP paths for $C_{2}$ (indicated by red arrows and blue arrows, respectively). Then, vehicle $C_{2}$ will choose one path randomly.

TSP strategy: When real-time average travel time $\langle T\rangle$ of each link can be acquired by ITS, drivers will choose the paths with global shortest total travel time. Obviously, TSP is a dynamic guidance strategy, since travel time of each link is changing with the traffic demand.

ECO routing strategy: In practice, the travel cost of drivers should include the time cost and fuel cost. With the development of ITS, the real-time fuel consumption of vehicles can be also recorded and shared. Then, the average travel time cost and fuel cost of each link $r$ are $a\langle T\rangle$ and $b\langle F\rangle$, respectively, where $\langle F\rangle$ represents the average fuel consumption of link, $a$ and $b$ are the value of time and fuel price, respectively. According to the feedback of average travel cost of all links, drivers will choose the paths with global minimal total travel cost. For comparison, the new strategy and TSP paths will be updated with the same period $T_{u}=4 T=80 \mathrm{~s}$.

Table 1. Parameters of CMEM.

\begin{tabular}{|c|c|c|c|c|c|}
\hline Parameter & Description & Values & Parameter & Description & Values \\
\hline$\xi$ & $\begin{array}{c}\text { Fuel-to-air mass } \\
\text { ratio }\end{array}$ & 1 & $q$ & Payload $(k g)$ & 200 \\
\hline$\kappa$ & $\begin{array}{l}\text { Heating value of } \\
\text { typical gasoline } \\
\text { fuel }(\mathrm{kJ} / \mathrm{g})\end{array}$ & 46 & $C_{d}$ & $\begin{array}{c}\text { Coefficient of } \\
\text { aerodynamic drag }\end{array}$ & 0.7 \\
\hline$\psi$ & $\begin{array}{c}\text { Conversion factor } \\
(g / s \text { to } L / s)\end{array}$ & 740 & $\rho$ & Air density $\left(\mathrm{kg} / \mathrm{m}^{3}\right)$ & 1.2041 \\
\hline$k$ & $\begin{array}{l}\text { Engine friction } \\
\text { factor }(\mathrm{kJ} / \mathrm{rev} / L)\end{array}$ & 0.25 & $A$ & $\begin{array}{c}\text { Frontal surface area } \\
\left(\mathrm{m}^{2}\right)\end{array}$ & 2 \\
\hline$N$ & $\begin{array}{c}\text { Engine speed } \\
(\mathrm{rev} / \mathrm{s})\end{array}$ & 40 & $g$ & $\begin{array}{c}\text { Gravitational constant } \\
\left(\mathrm{m} / \mathrm{s}^{2}\right)\end{array}$ & 9.81 \\
\hline V & $\begin{array}{c}\text { Engine } \\
\text { displacement }(L)\end{array}$ & 2 & $C_{r}$ & $\begin{array}{l}\text { Coefficient of rolling } \\
\text { resistance }\end{array}$ & 0.01 \\
\hline$\eta$ & $\begin{array}{c}\text { Efficiency } \\
\text { parameter for } \\
\text { engines }\end{array}$ & 0.9 & $\eta_{t f}$ & $\begin{array}{c}\text { Vehicle drive train } \\
\text { efficiency }\end{array}$ & 0.4 \\
\hline$\omega$ & Curb weight $(\mathrm{kg})$ & 1800 & & & \\
\hline
\end{tabular}

\section{Results}

In this section, we compare the effects of three route guidance strategies on the performance of traffic system on Manhattan-alike networks. The network size is set to $N=16$ and $L=$ 100 cells. The parameters of vehicle dynamic is $v_{\max }=3 \mathrm{cells} / \mathrm{s}$ and $p=0.1$. Assuming each cell corresponds to $7.5 \mathrm{~m}$ and each time step corresponds to 1 second, the maximum velocity corresponds to $81 \mathrm{~km} / \mathrm{h}$. Without losing generality, $a=0.01 R M B / \mathrm{s}$ and $b=$ $6.8 R M B / L$. We use closed boundary conditions to simulate traffic flow. i.e., vehicles are randomly assigned with their origin and destination. Once it arrives at the destination, another destination is reassigned to it. All simulation results are collected and averaged after the transient time.

Fig. 2 shows the average velocity $\langle V\rangle$ and average travel length $\langle L\rangle$ versus density $\rho$ for three routing strategies. In general, $\langle V\rangle$ decreases monotonically, and $\langle L\rangle$ rises monotonically with $\rho$. When $\rho$ is small, the system is in free flow state. For free flow, GSP gives a higher $\langle V\rangle$ and $\langle L\rangle$ lower than TSP and ECO-routing. Since GSP does not have a real-time information feedback, gridlock forms in the system and leads to the rapid drop of $\langle V\rangle$ with the increase of $\rho$. TSP and ECO-routing can dynamically update the paths, and help vehicles 
avoid traffic jams, leading to the better performance in the range of higher density. Furthermore, from the inset of Fig.2(a), it can be seen that TSP is slightly ahead of ECOrouting in terms of the average velocity $\langle V\rangle$, but has a larger $\langle L\rangle$ than ECO-routing.

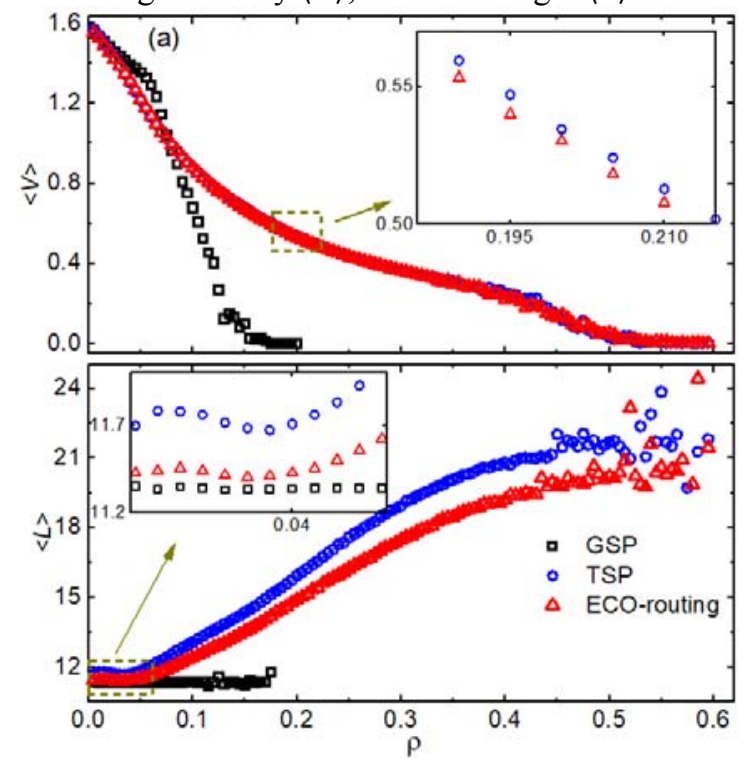

Fig. 2. (a)Average velocity $\langle V\rangle$ versus density $\rho$; (b) Average travel length $\langle L\rangle$ versus density $\rho$.

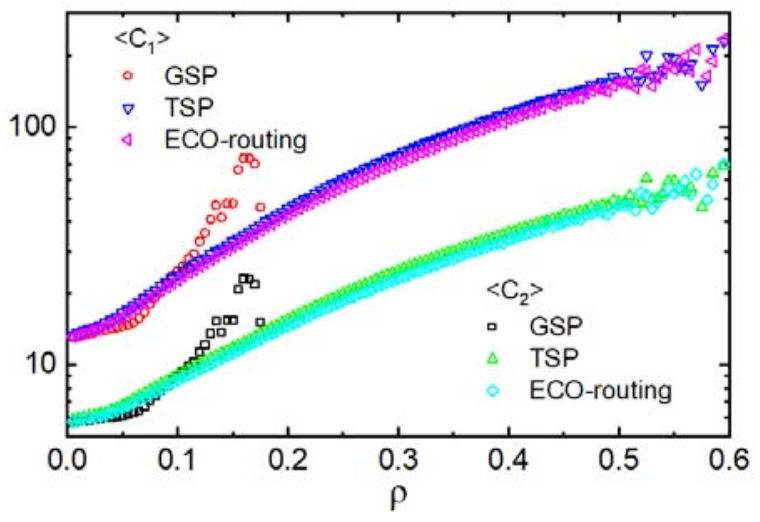

Fig. 3. Dash and solid lines are average fuel cost $\left\langle C_{2}\right\rangle$ and average total cost $\left\langle C_{1}\right\rangle$ versus density $\rho$, respectively.

In Fig.3, average travel total cost $\left\langle C_{1}\right\rangle$ and average fuel cost $\left\langle C_{2}\right\rangle$ per vehicle per arrival are plotted as a function of density $\rho$, respectively. Obviously, the height difference between two represents the average time cost. One can find that GSP shows the smallest $\left\langle C_{2}\right\rangle$ and $\left\langle C_{1}\right\rangle$ in the low density range, and ECO-routing performs better than TSP at all densities. This is because that the average travel length $\langle L\rangle$ of ECO-routing is smaller than that of TSP, as shown in Fig.2(b). The results indicate that our proposed ECO-routing strategy can help save the fuel consumption and reduce the travel cost, especially in high traffic level.

In Fig.4, we show the distribution of total travel cost for lanes when density $\rho=0.15$. Traffic jam emerges with GSP, and leads to very high travel cost in a wide area near the core. Both TSP and ECO-routing also show high travel cost in the center area. However, the number of lanes with high travel cost under TSP is smaller than ECO-routing. This is because that vehicles are distributed more evenly under TSP, and average velocity of TSP is larger 
than ECO-routing (see Fig.2(a)). The larger velocity indicates that vehicles move more freely, resulting in less fuel consumpion and shorter travel time. As a result, a lower average travel cost on lanes appears with TSP. Although the lane cost is higher for ECO-routing, the path length is shorter, thus the overall travel cost of ECO-routing is lower (see Fig.3).

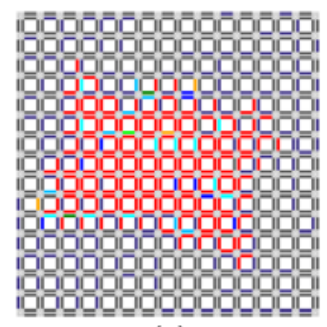

(a)

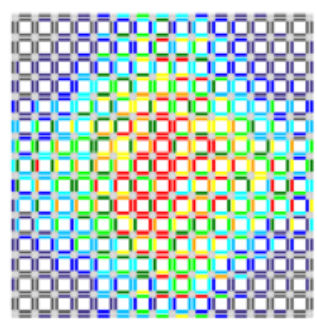

(b)

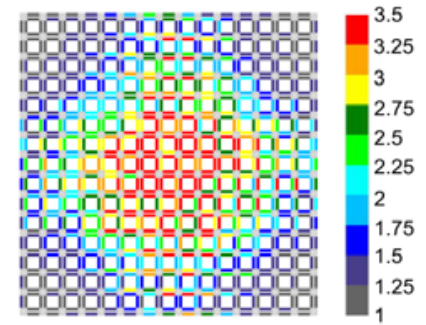

(c)

Fig. 4. Distribution of travel cost of all lanes for different routing strategies at density $\rho=0.15$ : (a) GSP; (b) TSP; (c) ECO-routing.

\section{Conclusion}

In this paper, we propose an advanced eco-routing strategy to reduce the fuel consumption of transportation system. Compared to the geographical shortest path strategy (GSP), the results show that the new strategy can improve the traffic capacity. Compared to the time shortest path strategy (TSP), the new strategy can efficiently save fuel consumption and travel cost by the less average travel length, since the unnecessary detours caused by information lag effect are avoided. To explain the phenomenon, the distribution of travel cost among all roads is also provided. Our research can be helpful to for the design of traffic navigation system in the future.

\section{Acknowledgement}

The research was supported by the National Natural Science Foundation of China (No.12072340, 11672289).

\section{References}

1. H. Li, A. Ravey, A. NDiaye, A. Djerdir, Energy Conversion and Management 192, 133 -149 (2019)

2. M. Li, Z. J. Ding, R. Jiang, M. B. Hu, B. H. Wang, Journal of Statistical Mechanics 12, P12001 (2011)

3. S. Darbha, S. Konduri, P. R. Pagilla, IEEE Transactions on Intelligent Transportation Systems 20 (5), 1954-1963 (2019)

4. Z. Zhong, E. E. Lee, M. Nejad, J. Lee, Transportation Research Part C 115, 102611 (2020)

5. T.Q. Tang, Q. Yu, K. Liu, Physica A 466, 1-9 (2017)

6. N. Cui, B. Chen, K. Zhang, Y. Zhang, X. Liu, J. Zhou, Physica A 513, 32-44 (2019)

7. Kai Nagel, Michael Schreckenberg, J. Phys. I France 2 (12), 2221-2229 (1992)

8. M. Barth, K. Boriboonsomsin, Transportation Research Record 2058 (1), 163-171 (2008)

9. W. Pan, Y. Xue, H.-D. He, W.-Z. Lu, Physica A 503, 154 - 162 (2018) 